opportunity for inconsistent application and limits the potential for replication and synthesis of evidence of effectiveness.

This study is the first step towards moving from general descriptions of implementation strategies to full descriptions of their active ingredients. This is essential to understand how strategies at an organisational and professional level can lead to observable changes in individual behaviour.

\section{P74 UNDERSTANDING THE IMPETUS FOR MAJOR SYSTEMS CHANGE: A MULTIPLE CASE STUDY OF DECISIONS AND NON-DECISIONS TO RECONFIGURE EMERGENCY AND URGENT CARE SERVICES}

${ }^{1} S$ McHugh*, ${ }^{2} E$ Droog, ${ }^{1} \mathrm{C}$ Foley, ${ }^{1} \mathrm{M}$ Boyce, ${ }^{2} \mathrm{O}$ Healy, ${ }^{1} \mathrm{~J}$ Browne. ${ }^{1}$ School of Public Health, University College Cork, Cork, Ireland; ${ }^{2}$ South/South West Hospital Group, Health Service Executive, Cork, Ireland

\subsection{6/jech-2019-SSMabstracts.225}

Background The optimal organisation of emergency and urgent care services (EUCS) is a perennial problem internationally. Similar to other countries, the Health Service Executive in Ireland pursued EUCS reconfiguration in response to quality and safety concerns, unsustainable costs and workforce issues. However, the implementation of reconfiguration has been inconsistent at a regional level. Our aim was to identify the factors that led to this inconsistency.

Methods Using a multiple case study design, case study regions were selected based on the extent of emergency department reconfiguration in the region (categorised as full, partial and little/no reconfiguration). Semi-structured interviews were conducted with a purposive sample of stakeholders who were centrally involved in the reconfiguration process in each region. Interview data were supplemented with documentary analysis of proposals for EUCS in each region. Data were analysed using a framework approach, drawing on an existing conceptual framework for major system change. Cross-case analysis was conducted iteratively to identify patterns and differences across the regions.

Results Six regions were selected for analysis and 42 interviews were analysed. The impetus to reconfigure ED services was triggered by patient safety events, and to a lesser extent by having a region-specific plan and an obvious starting point for changes. However, the complexity of the next steps and political influence impeded reconfiguration in several regions. Implementation was more strategic in regions that reconfigured later, facilitated by clinical leadership and 'lead-in time' to plan and sell changes.

Conclusion While the global shift towards centralisation of EUCS is driven by universal challenges, decisions about when, where and how much to implement are influenced by local drivers including context, people and politics. This can contribute to a public perception of inequity and distrust in proposals for major systems change.

\section{P75 EXAMINING TOTAL AND DOMAIN-SPECIFIC SEDENTARY BEHAVIOUR USING THE SOCIO-ECOLOGICAL MODEL - A CROSS-SECTIONAL STUDY OF IRISH ADULTS}

H Nicolson*, Darker, Hayes. Public Health and Primary Care, Trinity College Dublin, Dublin, Ireland

10.1136/jech-2019-SSMabstracts.226
Background Sedentary behaviour has been linked with detrimental effects on morbidity and mortality. This study aims to identify the individual, social and environmental correlates of total sedentary behaviour as well as across the contexts that sitting time accumulates in an Irish adult cohort.

Methods Cross-sectional analysis of data from 7,305 adults of the nationally representative Healthy Ireland Survey. Multivariate regression analyses were used to examine participants' socio-demographic characteristics, lifestyle factors, workplace activity patterns, physical and mental health status, and environmental factors, and their association with participants' total daily sitting times and sitting times across the domains of work, travel, leisure and screen-time.

Results Overall median of sitting time per day was 360 minutes (6 hours). Workplace sitting was the strongest predictor of sedentary behaviour. Male gender, higher education attainment, higher socio-economic classification and living in an urban dwelling were all associated with increased totaland occupational- sitting time $(\mathrm{p}<0.05)$. Insufficient physical activity levels was also associated with total sitting time $(p<0.001)$. Male gender, lower education attainment, a possible mental health problem, smoking and insufficient physical activity were all associated with increased screen-time sitting $(p<0.05)$. Higher education attainment, physical illness, a possible mental health problem, alcohol consumption and lower perceived neighbourhood attributes were all associated with higher transportation/leisure sitting times $(\mathrm{p}<0.05)$. Variance of the multivariate model for occupational sitting was $39.0 \%$ and $25.8 \%$ for total sitting.

Conclusion Having a sedentary occupation was the strongest predictor of sitting time in this population. The results of this study provide a starting position for the development of targeted interventions aimed at the most sedentary groups, such as professional and higher educated males with sedentary occupations.

\section{\begin{tabular}{|l|l}
\hline P76 SECULAR TRENDS AND COSTS OF MANAGEMENT OF \\
\hline
\end{tabular} ACUTE MYELOID LEUKAEMIA: EVIDENCE FROM POPULATION-BASED CANCER REGISTRATION DATA}

${ }^{1} \mathrm{AO}$ Ceilleachair*, ${ }^{2} \mathrm{M}$ Cahill, ${ }^{3} \mathrm{R}$ McMorrow, ${ }^{3} \mathrm{C}$ Donnelly. ${ }^{1}$ School of Public Health, UCC, Cork, Ireland; ${ }^{2}$ Haematology Dept., Cork University Hospital, Cork, Ireland; ${ }^{3}$ National Cancer Registry Ireland, Cork, Ireland

10.1136/jech-2019-SSMabstracts.227

Background Acute myeloid leukaemia (AML) is an aggressive blood cancer that, left untreated, proves fatal within a short period. Though numbers diagnosed annually are relatively small, treatment costs from induction therapy through to clinical remission potentially are in excess of $€ 200,000$. We present, for the first time, using cancer registration data, evidence on trends in the incidence of AML for Ireland, together with an assessment of the costs of manging the disease.

Methods Cancer registration data on individuals aged 20 years and older diagnosed with AML (ICD-10 C92.0) 1994-2013 were extracted from a population register. EASR and crude incidence rates were calculated with $95 \%$ confidence intervals by five-year age bands. Cases were assigned to one of four treatment pathways on the basis of patient characteristics. These were an intensive chemotherapy pathway, a pathway with bone marrow transplantation, a low intensity chemotherapy pathway and a best supportive care pathway. Resource use for each pathway was determined using clinical guidelines, 